\title{
Fast Pyrobolometers for Measurements of Flasma Heat Fluxes and Radiation Losses in the MST Reversed Field Pinch.
}

\author{
G. Fiksel, J. Frank, and D. Holly. \\ Department of Physics, University of Wisconsin - Madison \\ Madison, WI 53706
}

\begin{abstract}
Two types of fast bolometers are described for the plasma energy transport study in the Madison Symmetric Torus plasma confinement device. Both types use pyrocrystals of $\mathrm{LiTaO}_{3} \mathrm{o}_{2} \mathrm{LiNbO}_{3}$ as the sensors. One type is used for measurements of the radiated heat losses and is situated at the vacuum shell inner surface. Another type is insertable in the plasma and measures the plasma particle heat flux. The frequency response of the bolometers is measured to be in the $150-200 \mathrm{kHz}$ range. The range of the measured power fluxes is $0.1 \mathrm{~W} / \mathrm{cm}^{2}$ $10 \mathrm{~kW} / \mathrm{cm}^{2}$ and can be adjusted by changing the size of the entrance aperture. The lower limit is determined by the amplifier noise and the frequency bandwidth, the higher limit by destruction of the bolometer sensor.
\end{abstract}

MASTER 


\section{INTRODUCTION}

One of the directions of the MST ${ }^{1}$ (Madison Symmetric Torus) program is the study of the plasma turbulence and transport and their effect on the plasma confinement. MST is a Reversed Field Pinch ( RFP) plasma confinement device with major radius $1.5 \mathrm{~m}$, and minor radius $0.52 \mathrm{~m}$. The range of the plasma parameters is: plassma current Ip $=150-600 \mathrm{kA}$, plasma density $n_{\mathrm{e}}=0.8 \times 10^{13}$ $2 \times 10^{13} \mathrm{~cm}^{-3}$, electron and ion temperatures $T_{e}$ and $T_{i}$ up to $500 \mathrm{eV}$, and plasma duration time up to $80 \mathrm{~ms}$.

The plasma particle and energy transport in the MST is anomalously high, ${ }^{2}$ presumably due to fluctuations of electrical and magnetic fields. The magnetic field fluctuations can drive a radial energy transport due to a correlation between fluctuations of the heat flux $\tilde{q}_{\|}$parallel to the magnetic field and fluctuations of the radial component of the magnetic field $\tilde{B}_{r}$. The expression for the radial energy transport due to the magnetic field fluctuations is 2,3

$$
q_{r}=\frac{\left\langle\tilde{q}_{\|} \tilde{B}_{r}\right\rangle}{B_{0}}
$$

where $q_{r}$ is the radial heat flux, and $B_{0}$ is the total magnetic field. The brackets denote the time average.

The fluctuations of the magnetic field components in the MST have been directly measured by various kinds of insertable magnetic coils 4 . The magnetic field fluctuations exhibit a broad band frequency character (in the $100 \mathrm{kHz}-200 \mathrm{KHz}$ range) and the amplitude of the fluctuations is high $\left(B_{r} / B_{0} \approx i-2 \%\right)$. An estimate of the radial heat flux with Eq. 1 using a reasonable estimation of the parallel heat 
flux gives a value of the heat flux high enough to account for the total energy transport. For example, assuming $\tilde{q}_{\|}=1 \mathrm{~kW} / \mathrm{cm}^{2}$ and $\quad \tilde{B}_{r} / B_{0}=0.01$, yields $q_{r}$ $=10 \mathrm{~W} / \mathrm{cm}^{2}$ provided there is a perfect correlation between fluctuations of the radial magnetic field and the parallel heat flux. The total rate of the energy losses in the MST is of the same order.

Nevertheless, no direct measurements of the plasma parallel heat flux have been available to study this mechanism of the energy transport. Therefore, our aim was to create an insertable bolometer for direct measurements of the plasma parallel heat flux. The expected amplitude of the plasma parallel heat flux is of the order of $100 \mathrm{~W} / \mathrm{cm}^{2}-10 \mathrm{~kW} / \mathrm{cm}^{2}$; the expected frequency range of the oscillations is in the $200 \mathrm{kHz}$ range. Aside from the transport study an insertable bolometer is an extremely useful plasma diagnostic tool, allowing one to measure directly the heat fluxes inside a plasma and complementing, therefore, Langmuir probes and miniature energy analyzers.

Another problem, linked to the energy transport study, is the measurement of the radiated power. Currently, the radiated power is measured by a platinum resistive bolometer ${ }^{5}$. The frequency bandwidth of the bolometer is limited by its thermal inertia and is of the order of $2-3 \mathrm{kHz}(100 \mu \mathrm{s}$ rise time). To adequately study the fluctuation induced transport we needed a bolometer with a broader bandwidth.

\section{PYROELECTRIC BOLOMETER}

The requirements that the bolometer must meet are outlined below :

- Broad frequency bandwidth $(100-200 \mathrm{kHz})$. 
- Plasma and vacuum environment compatibility (vacuum materials, absence of impurities, contamination and/or outgassing).

- Ability to withstand high heat load.

- Immunity to the plasma electrostatic and magnetic pickup noise A bolometer based on the principle of pyroelectricity ${ }^{6,7}$ meets all these requirements. Pyroelectric materials are characterized by a permanent polarization which varies with the temperature, causing the polarization charges to flow in an external circuit. Since this polarization response time is very short ${ }^{8}$ (in the picoseconds range) this effect can be utilized for very fast detection of power fluxes. Pyroelectric bolometers have been extensively used in various applications ranging from detection of pulsed laser radiation ${ }^{8}$ to measurements of radiation losses in plasma devices $9-10$.

Figure 1 shows a schematic of the insertable pyroelectric bolometer for plasma flux measurements. The heart of the bolometer is a pyrocrystal of $\mathrm{LiNbO}_{3}$ or $\mathrm{LiTiO}_{3}$. The choice of the pyrocrystals was based on their high pyroelectric sensitivity, high Curie point ( the temperature at which the spontaneous polarization disappears), which is $610^{\circ} \mathrm{C}$ for $\mathrm{LiTaO}_{3}$ and $1150{ }^{\circ} \mathrm{C}$ for $\mathrm{LiNbO}_{3}$ and their good mechanical, thermal and vacuum properties. The diameter of the crystals is $1 \mathrm{~cm}$ and the thickness is $1 \mathrm{~mm}$. Both sides of the crystals are metallized with $5 \mu \mathrm{m}$ of gold over $200 \AA$ of $\mathrm{Cr}$. The crystals were fabricated and plated by DELTRONIC Crystal Industries, Inc., Dover, NJ. The crystals proved to be very stable, rigid, and reliable.

The copper shell, which is electrically grounded, serves as a bolometer case. The crystal is slightly recessed in the insulator and pressed against the shell with the help of the springs. This enables the front face of the crystal to be in a good and 
stable electrical contact with the grounded copper shell forming a good electrostatic and electromagnetic shield. The back face of the crystal is connected to a current-to-voltage converter and the pyroelectric current is measured. The copper shell is enclosed in a boron nitride shroud which forms a protective shield against the destructive plasma power. A small part of this power enters the bolometer through a small aperture in the boron nitride cover.

Typically, the bolometer was inserted into the plasma and aligned along the magnetic field so it measured the power flowing along the magnetic field line. The bolometer is two-sided, which allows one to measure power fluxes simultaneously from two opposite directions. The net power flux was taken as the difference between the two signals. This approach allows one, in addition, to subtract the plasma noise signals (they have been found to be small) and the radiation power (also found to be small in comparison to the direct plasma power flux).

A repeller electrode is located between the entrance aperture and the crystal. The electrode is biased negatively with respect to the ground and repels plasma electrons which have energies less than the biasing voltage. The purpose of this electrode is twofold. First, by scanning the voltage it is possible to measure the plasma electron energy distribution function. Second, this electrode can be used to gate the bolometer. The negative voltage is held high enough, usually $-1 \mathrm{kV}$, to prevent almost all the electrons from entering the bolometer. At a given moment the voltage is shorted to zero for a short period of time, usually 1-2 ms, and during this time the energy flux is being measured. This gating decreases the total energy deposited into the bolometer, so the bolometer can be inserted deeper into the plasma without damage. 
The bolometer for measurements of the radiated power is stationary, located at the inner surface of the vacuum shell. The design of the radiation bolometer is very similar to the design of the insertable bolometer except that the radiation bolometer is one-sided, has a bigger entrance aperture and does not feature the repeller electrode. In addition, we use $\mathrm{LiTaO}_{3}$ for the radiation bolometer, while for the insertable bolometer $\mathrm{LiNbO}_{3}$ was used because of its higher Curie point.

\section{MEASUREMENTS AND CALIBRATION}

It can be shown $\mathrm{n}^{6}$, that under some conditions the pyroelectric current is proportional to the incident power:

$$
I=\alpha \frac{\gamma}{c \rho \delta} Q
$$

where $Q$ is the incident power, $\gamma$ is the pyroelectric coefficient, $c$ and $\rho$ are the specific heat and density, $\delta$ is the thickness of the crystal, and $\alpha$ is the energy reflection coefficient. The conditions under which $\mathrm{Eq}(2)$ is valid consist of limiting the characteristic time $\tau$ of the heat flux from the fast and from the slow side:

$$
\tau_{a b s}, R_{L} C<<\tau<<\tau_{c r}
$$

where $\tau_{a b s}=c_{a b s} \rho_{a b s} K_{c b s}^{-1} \delta_{a b s}^{2}=k_{a b s}^{-1} \delta_{a b s}^{2}$ and $\tau_{c r}=c_{c r} \rho_{c r} K_{c r}^{-1} \delta_{c r}^{2}=k_{c r}^{-1} \delta^{2}$ are the temperature diffusive times in the absorbent layer and the crystal correspondingly. Here, $K$ is the heat conductivity and $k$ is the temperature diffusivity of the materials. The electrical time constant $R_{L} C$ is the product of the 
load resistance and the crystal capacitance. It is clear that a thinner absorption layer provides a faster response time. Choosing $5 \mu \mathrm{m}$ of gold as the absorbing layer results in $\tau_{a b s}=0.25 \mu \mathrm{s}$. This thickness also provides complete absorption of $\mathrm{UV}$ and soft $\mathrm{X}$-ray radiation as well as the plasma electrons with the energies below $10 \mathrm{keV}$. The capacitance of the crystal and accompanying wiring is $C=500$ $\mathrm{pF}$, which together with $R_{L}=1 \mathrm{k} \Omega$ gives $R_{L} C=0.5 \mu \mathrm{s}$. On the other side, the temperature diffusive time for a $1 \mathrm{~mm}$ thick crystal is about $1 \mathrm{sec}$. Therefore, the expected time response of the bolometer is from $1 \mu \mathrm{s}$ to $1 \mathrm{sec}$.

Figure 2 shows the schematics and results of the bolometer time response measurements. The bolometer was illuminated with a $\mathrm{CO}_{2}$ laser. The laser beam was passing through a Bragg cell and the passing deflected beam power was modulated by modulating the radiofrequency drive voltage to the cell. The pyroelectric current was measured with a current-to-voltage converter with the gain of $10^{6} \mathrm{~V} / \mathrm{A}$ and bandwidth of $350 \mathrm{kHz}$. The upper trace shows the modulating voltage and the lower trace shows the pyroelectric response. The shown signals are averaged over several traces in order to decrease the bolometer amplifier noise. The measured rise time is $\tau_{10-90 \%}=1.9 \mu \mathrm{s}$ (170 kHz bandwidth). This time agrees well with a convolution of the laser power rise time (measured at $0.3 \mu \mathrm{s}$ ), the absorption layer temperature diffusion time- $\tau_{a b s}=0.25 \mu \mathrm{s}, R_{L} C=$ $0.5 \mu \mathrm{s}$ and the amplifier rise time $1.2 \mu \mathrm{s}$.

The absolute sensitivity of the bolometer was calculated using the crystals technical data and calibrated in situ by comparison with the absolutely calibrated platinum resistive bolometer installed in MST. For a $\mathrm{LiTaO}_{3}$ crystal with thickness $\delta=0.1 \mathrm{~cm}$ and $\gamma=0.019 \mu \mathrm{C} / \mathrm{cm}^{2}{ }^{\circ} \mathrm{C}($ Ref.11 $), c=0.43 \mathrm{~J} / \mathrm{g}^{\circ} \mathrm{C}, \rho=7.45$ $\mathrm{g} / \mathrm{cm}^{3}$ the theoretical bolometer response (Eq. 2) is $5.9 \times 10^{-8} \mathrm{~A} / \mathrm{W}$, assuming the 
absorption to be $100 \%$. Figure 3 shows the results of the measurements of radiated power in the MST. The output of the pyrobolometer was calculated using the theoretical response and compared with the output of the platinum resistive bolometer. The agreement is very good. Since the radiated power is concentrated mostly in the $\mathrm{UV}$ anci soft $\mathrm{X}$-ray region, then the assumption of $100 \%$ absorption is correct. The same experiment was done with $\mathrm{LiNBO}_{3}$ crystals and, again, the output of the platinum resistive bolometer was identical to the output of the pyrobolometer with the response of $1.8 \times 10^{-8} \mathrm{~A} / \mathrm{W}$. Based on published $\mathrm{LiNbO}_{3}$ pyroelectric coefficients ${ }^{12},{ }^{13}$ the expected response is $1.4 \times 10^{-8}$ $\mathrm{A} / \mathrm{W}-2.2 \times 10^{-8} \mathrm{~A} / \mathrm{W}$.

Figure 4. shows results of the plasma flux measurements. The gate was modulated with $2 \mathrm{~ms}$ long square $1 \mathrm{kV}$ pulses. The plot shows a great difference between the heat flux parallel and antiparallel to the magnetic field. The plasma interference is very small. We want to emphasize that the gating of the bolometer is absolutely necessary if it is to be inserted deep into a plasma. Overexposing of the bolometer causes overheating of the crystal surface and/or evaporation of the metal plating or depolarization. The surface temperature $T$ depends on the incident power density $q$ as 8

$$
T=\frac{q}{c \rho} \sqrt{\frac{t}{\pi k}}
$$

where $t$ is the power pulse time duration and $k$ is the crystal temperature diffusivity. We assumed here that $\tau_{a b s} \ll \tau \ll<\tau_{c r}$. Using $T=1000^{\circ} \mathrm{C}$ as the temperature threshold and $k=10^{-2} \mathrm{~s} \mathrm{~cm}^{-2}$ gives a power threshold condition

$q \sqrt{t}=500 \mathrm{~W} \mathrm{~cm}^{-2} \mathrm{~s}^{1 / 2}$. 
For the plasma pulse duration $t=0.05 \mathrm{sec}$ the power density threshold is $\approx 2 \mathrm{~kW}$ $\mathrm{cm}^{-2}$. Gating with $t=0.002 \mathrm{sec}$ raises the power density threshold up to $10 \mathrm{~kW}$ $\mathrm{cm}^{-2}$. We experimentally observed that for an ungated bolometer the gold layer is burned through at a power density level of about $2 \mathrm{~kW} \mathrm{~cm}^{-2}$. The size of the damaged spot is equal to the entrance aperture size which is natural since the plasma electrons carrying the bulk of the heat are tied to the magnetic field lines. Immediately after a damage the plasma high frequency noise interference significantly increases, making fluctuation measurements impossible. It is interesting to notice that an integrated output, corresponding to the low frequency part of the plasma heat flux, still remains unchanged.

The stu:dy on the magnetic fluctuation induced radial energy transport is ongoing.

\section{SUMMARY AND CONCLUSIONS.}

We have designed and fabricated fast pyroelectric bolometers for the ongoing study of the plasma transport in the MST. Two types of bolometers have been designed - an insertable bolometer for measurement of the direct plasma heat flux, and a radiated bolometer, situated outside of the plasma, for measurements of the radiation heat losses. The bolometers use pyrocrystals of $\mathrm{LiNbO}_{3}$ and $\mathrm{LiTaO}_{3}$ as the sensors.

The bandwidth of the bolometer is measured at $150-200 \mathrm{kHz}$, which allows one to study the transport induced by fast fluctuations. The dynamic range of the bolometers is of 
$0.1 \mathrm{~W}-10 \mathrm{~kW}$ and can be adjusted by changing the size of the entrance aperture.

Several features are implemented to increase the bolometer ability to withstand a high plasma heat load. First, the bolometer is enclosed in a boron nitride shroud. Second, the pulsed repeller drastically decreases the total amount of deposited energy.

\section{ACNOWLEDGMENTS}

The authors appreciate assistance of Jeff Bruner, DELTRONIC.

The authors wish to thank Michael Reyfman and Michael Borchardt for technical support.

The work was supported by the US Department of Energy. 


\section{List of References}

1 R.N. Dexter, D.Kerst, T.W. Lovell, S.C. Prager, and J.C. Sprott, Fusion Technol. 19, 131 (1991).

2 S.C. Prager, Plasma Phys. Controlled Fusion, 32, 903 (1990).

3 T.D. Rempel, A.F. Almagri, S. Assadi, D.J. Den Hartog, S.A. Hokin, S.C. Prager, J.S. Sarff, W. Shen, K.L. Sidikman, C.W. Spragins, J.C. Sprott, M.R. Stoneking, and E.J. Zita, Phys. Fluids B 4, 2136 (1992).

4 A.F. Almagri, S.Assadi, S.C. Prager, J.S. Sarff, and D.W. Kerst, Phys. Fluids B 4, 4080 (1992).

5G. Miller, J.C. Ingraham, and L.S. Schrank, Rev. Sci. Instrum. 59, 700 (1988).

6 J. Cooper, J. Sci. Instrum, 39, 467 (1962).

7E.L. Dereniak and D.G. Crow, Optical Radiation Detectors, John Wiley, New York.

8 C.B. Roundy, R.L. Byer, D.P. Phillion, and D.J. Kuizenga, Optics

Communications, 10, 374 (1974).

${ }^{9}$ A.N. Vertiporokh, S.Yu. Lukyanov, and Yu.S. Maksimov, Sov. J. Plasma Phys. 8, 243 (1982).

10E.D. Andryukhina, A.N. Vertiporokh, K.S. Dyablin, Yu.S. Maksimov, O.I.

Fedyanin, and I.S. Shpigel, Sov. J. Plasma Phys. 7, 27 (1981).

11A.M. Glass, Appl. Phys. Letters, 13, 147 (1968).

12V.V. Lemanov and B.V. Sukharev, Sov. Tech. Phys. Lett, 9, 218 (1983).

${ }^{13}$ C.H. Balmer and W.K. Burns, Appl. Phys. Lett. 48, 21 (1986). 


\section{List of Figures.}

Figure 1. Schematic of insertable bolometer.

Figure 2. Bolometer time response calibration.

Oscilloscope traces: Ch 1- Bragg cell modulating signal, Ch 2 - pyrobolometer response.

Figure 3. Radiation power measurements.

Upper curve - Ohmic power. Lower curve - radiation power: solid line pyrobolometer signal, dashed line - resistive bolometer signal.

Figure 4. Darallel heat flux measurements.

Parallel to B signal shows measured heat flux when the bolometer is facing fast electrons. Antiparallel to B signal corresponds to the bolometer facing opposite direction. 


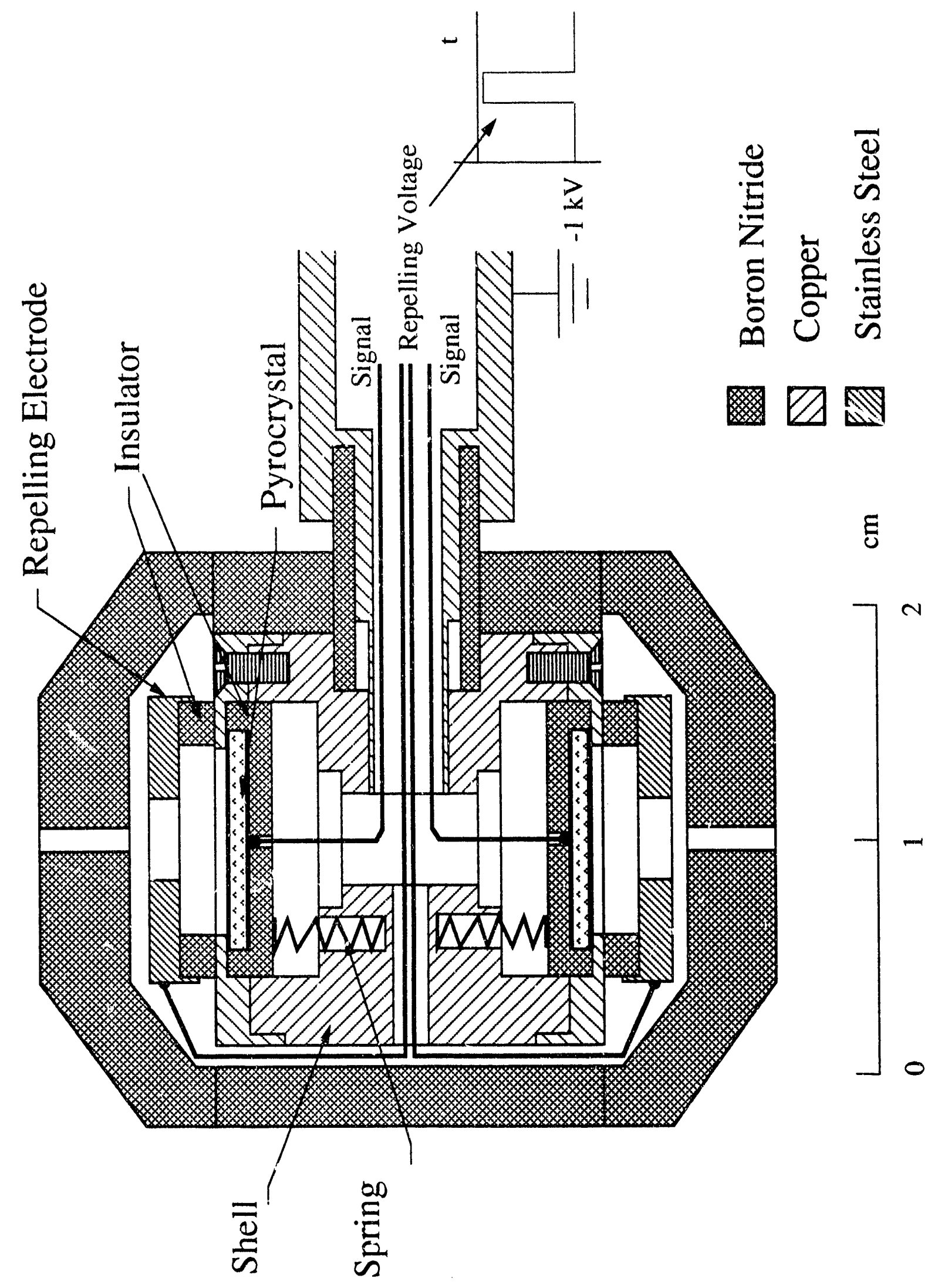



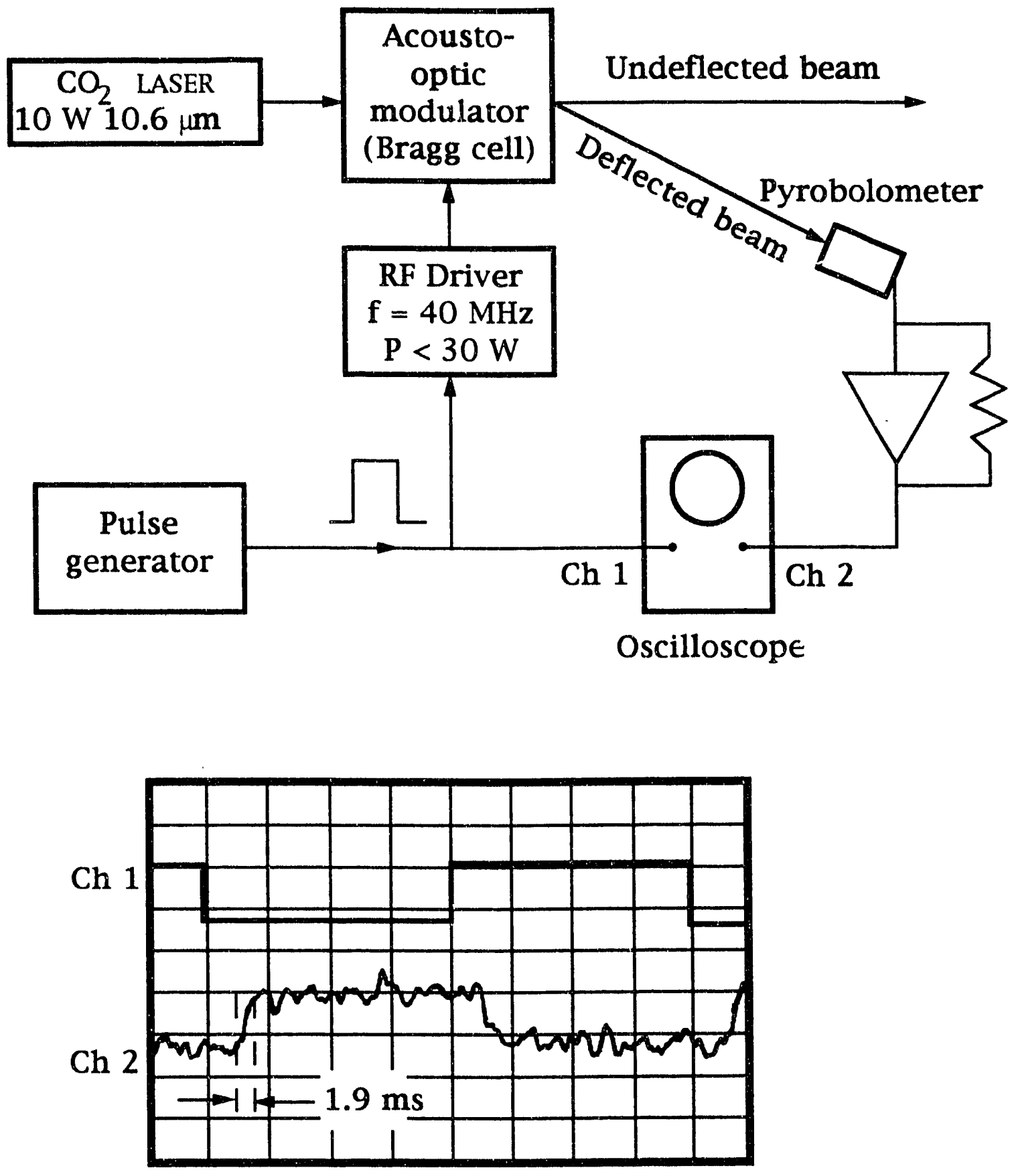

Time $(5 \mu \mathrm{s} / \mathrm{div})$

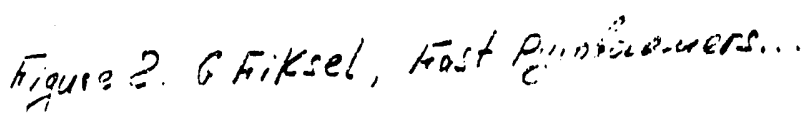




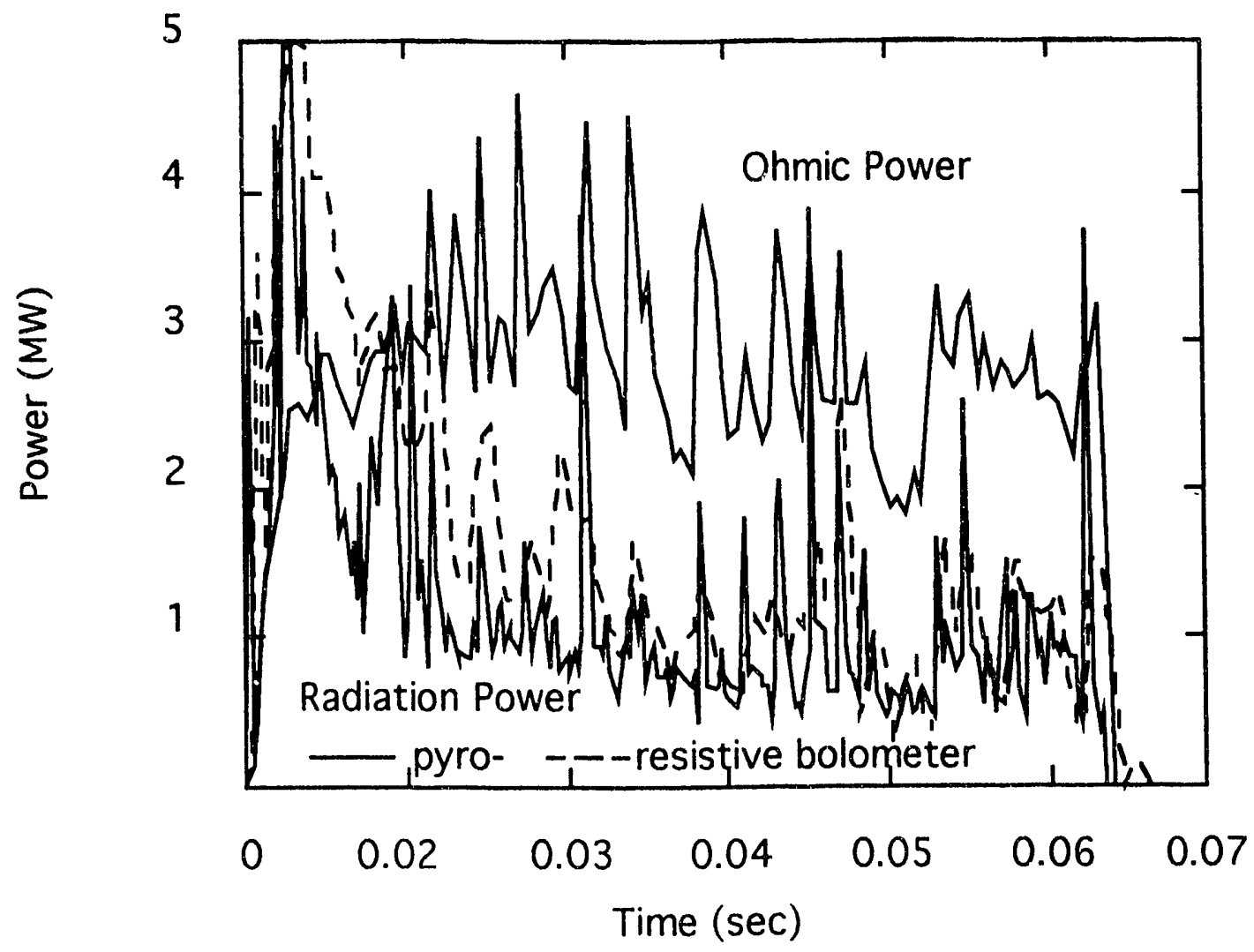

Fioure 3. G. Fiksel, hast uirobolemeters... 


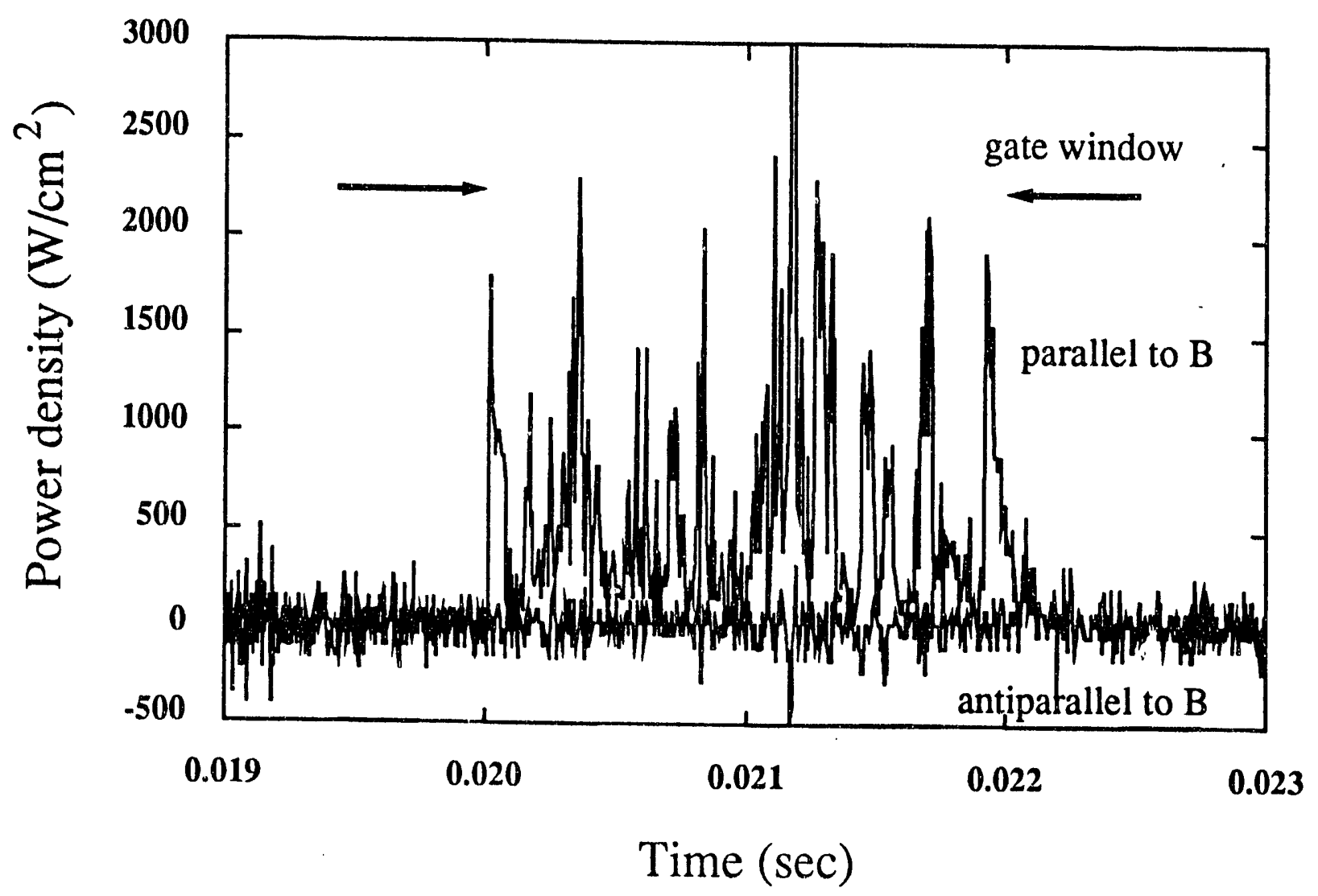

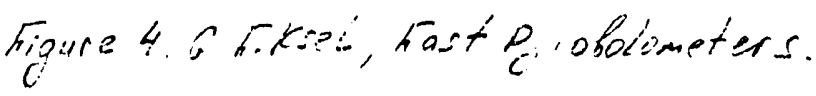




\section{EXTERNAL DISTRIBUTION IN ADDITION TO UC-20}

S.N. Rasband, Brigham Young University

J.B. Taylor, Institute for Fusion Studies, The University of Texas at Austin

M.A. Abdou, University of California, Los Angeles

R.W. Conn, University of California, Los Angeles

T. Dolan, INEL

R. Smith, University of lowa

F.W. Perkins, PPPL

O. Ishihara, Texas Technical University

P.E. Vandenplas, Association Euratom-Etat Belge, Belgium

Centro Brasileiro de Pesquisas Firicas, Brazil

P. Sakanaka, Institute de Fisica-Unicamp, Brazil

Mme. Monique Bex, GANIL, France

J. Radet, CEN/CADARACHE, France

University of loannina, Greece

S. Ortolani, Istituto Gas Ionizzati, EURATON-ENEA-CNR Association, Italy

R. Andreani, Associazione EURATOM-ENEA sulla Fusione, Italy

Y. Kondoh, Gunma University, Kiryu, Gunma, Japan

H. Toyama, University of Tokyo, Japan

FOM-Instituut voor Plasmafysica "Rijnhuizen", The Netherlands

Z. Ning, Academia Sinica, People's Republic of China

P. Yang, Shandong University, People's Republic of China

S. Zhu, University of Science \& Technology of China, People's Republic of China

I.N. Bogatu, Institute of Atomic Physics, Romania

M.J. Alport, University of Natal, Durban, South Africa

R. Storer, The Flinders University of South Australia, South Australia

B. Lehnert, Royal Institute of Technology, Sweden

Librarian, CRPP, Ecole Polytechnique Federale de Lausanne, Switzerland

B. Alper, Culham Laboratory, UK

A. Newton, Culham Laboratory, UK

6 for Chicago Operations Office

4 for individuals in Washington Offices

INTERNAL DISTRIBUTION IN ADDITION TO UC-20

80 for local group and file 

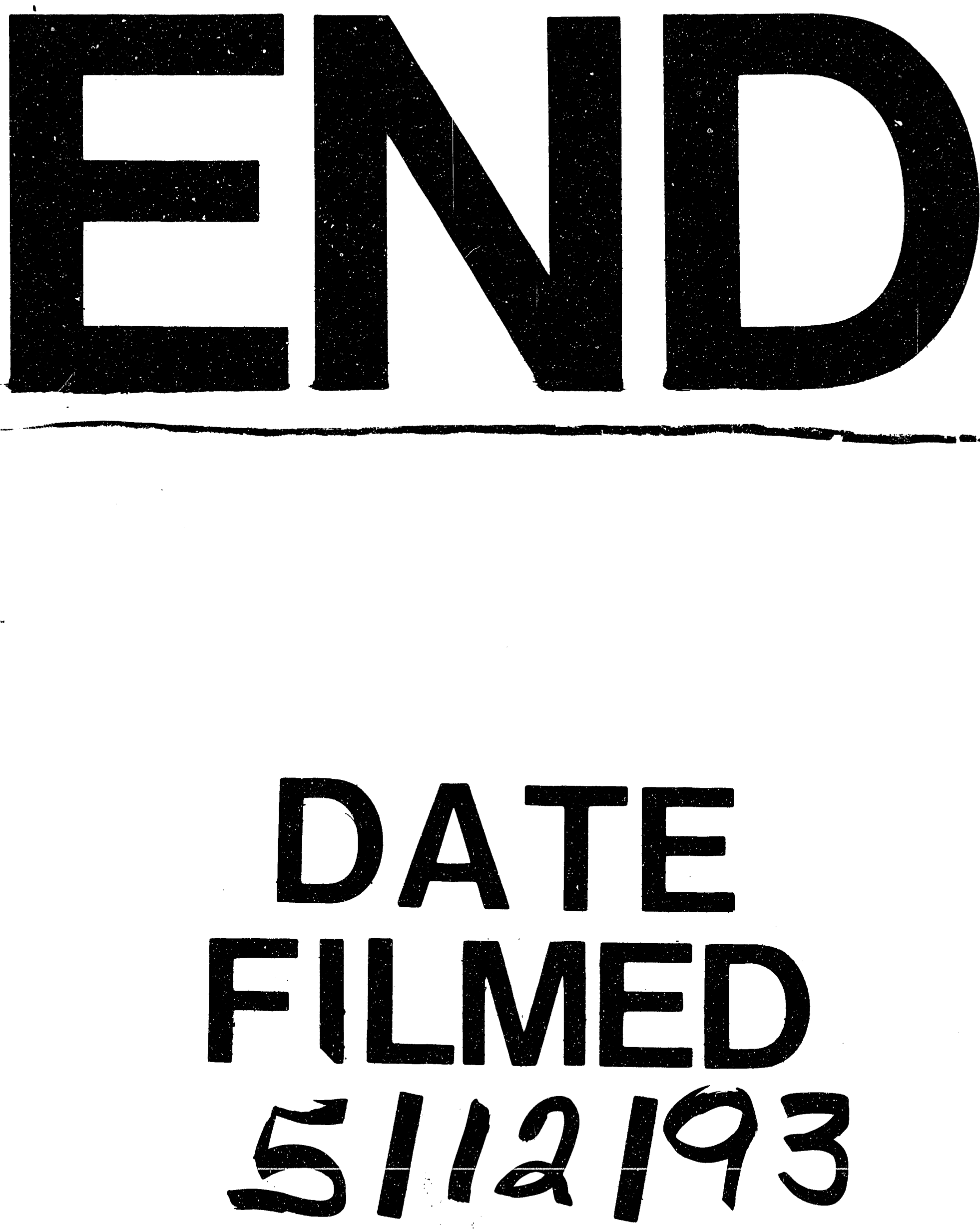
